\title{
LCMV Beamforming for a Novel Wireless Local Positioning System: Nonstationarity and Cyclostationarity Analysis
}

\author{
Hui Tong, Jafar Pourrostam, and Seyed A. Zekavat \\ Department of Electrical and Computer Engineering, Michigan Technological University, 1400 Townsend Drive,
} Houghton, MI 49931, USA

Received 24 June 2006; Revised 29 January 2007; Accepted 21 May 2007

Recommended by Kostas Berberidis

This paper investigates the implementation of a novel wireless local positioning system (WLPS). WLPS main components are: (a) a dynamic base station (DBS) and (b) a transponder, both mounted on mobiles. The DBS periodically transmits ID request signals. As soon as the transponder detects the ID request signal, it sends its ID (a signal with a limited duration) back to the DBS. Hence, the DBS receives noncontinuous signals periodically transmitted by the transponder. The noncontinuous nature of the WLPS leads to nonstationary received signals at the DBS receiver, while the periodic signal structure leads to the fact that the DBS received signal is also cyclostationary. This work discusses the implementation of linear constrained minimum variance (LCMV) beamforming at the DBS receiver. We demonstrate that the nonstationarity of the received signal causes the sample covariance to be an inaccurate estimate of the true signal covariance. The errors in this covariance estimate limit the applicability of LCMV beamforming. A modified covariance matrix estimator, which exploits the cyclostationarity property of WLPS system is introduced to solve the nonstationarity problem. The cyclostationarity property is discussed in detail theoretically and via simulations. It is shown that the modified covariance matrix estimator significantly improves the DBS performance. The proposed technique can be applied to periodic-sense signaling structures such as the WLPS, RFID, and reactive sensor networks.

Copyright (c) 2007 Hui Tong et al. This is an open access article distributed under the Creative Commons Attribution License, which permits unrestricted use, distribution, and reproduction in any medium, provided the original work is properly cited.

\section{INTRODUCTION}

This paper investigates how to implement optimal beamforming for a novel wireless local positioning system (WLPS). We focus on how to estimate covariance matrix for optimal beamforming, because the specific signaling scheme in this WLPS, that is, cyclostationarity, enables a novel covariance matrix estimator.

The WLPS consists of two main components [1]: a dynamic base station (DBS) and a transponder (or possibly a number of transponders), all mounted on mobiles. The DBS periodically transmits ID request signals (a short burst of energy). Each time a transponder detects the ID request signal, it sends its unique ID (a signal with a limited duration) back to the DBS. In the WLPS, the DBS detects and tracks the positions and IDs of the transponders in its coverage area. The position of a transponder is determined by the combination of time-of-arrival (TOA) and direction-of-arrival (DOA). TOA is estimated via the time difference between the transmission of ID request signal and the reception of the corresponding ID. DOA estimation would be possible if an antenna array is installed at the DBS receiver [2].
In WLPS, a single unit (the DBS) is capable of positioning transponders located in its coverage area. In systems such as cell phone positioning [3] and radio frequency ID [4], multiple units should cooperate in the process of positioning. Accordingly, the WLPS has many civilian and military applications. For example, in vehicle collision avoidance applications, each vehicle (car) may carry a DBS and each pedestrian may carry a transponder. Then, each vehicle is able to position (and identify) pedestrians. Another possible application of the WLPS is airport security, where security guards may carry DBSs and passengers may carry transponders.

The WLPS can be considered as a merger of positioning and communication systems. The TOA/DOA estimation is the primary procedure for positioning, while the ID detection process is supported by communications. This paper investigates the ID detection performance, that is, the communication aspect of the WLPS, while the TOA/DOA estimation process is discussed in $[5,6]$.

As depicted in [7], the main source of error in the ID detection process is the interference from other transponders. To reduce this interference, direct sequence code division multiple access (DS-CDMA) and beamforming techniques 
are adopted in the WLPS. The conventional beamforming methods (delay and sum) in the WLPS have been discussed in [7]. In general, linear constrained minimum variance (LCMV) beamforming outperforms conventional beamforming in terms of interference suppression [8]. Therefore, it is natural to extend our study from conventional beamforming to LCMV beamforming.

An important step to perform LCMV beamforming is the estimation of the covariance matrix of the received signal. Considering stationary signals, sample covariance accurately estimates the true signal covariance [9]. However, in the WLPS, the received signal at the DBS receiver is not stationary, because the DBS transmits ID request signals noncontinuously. The nonstationarity of the received signal causes the sample covariance to be an inaccurate estimate of the true signal covariance. The errors in this covariance estimate limit the applicability of LCMV beamforming in the WLPS.

In this work, a modified covariance matrix estimator is proposed. The transponders transmit signals noncontinuously and repetitively. Accordingly, the DBS received signal is nonstationary and cyclostationary. The proposed modified covariance matrix estimator exploits the cyclostationarity to counter the nonstationarity problem. A detailed theoretical analysis shows that, in most practical situations, the cyclostationarity duration is sufficiently long to ensure an accurate estimate. Finally, the WLPS ID detection performance is numerically simulated. The numerical results confirm that the modified covariance matrix estimator improves the WLPS performance significantly. It should be further noted that the proposed estimator is not restricted to this particular WLPS system: it is possible to apply this estimator to any system that exhibits repetitive structures. Hence, the proposed covariance matrix estimator has a wide range of applications.

Beamforming [10] and cyclostationarity [11] have been studied separately for more than fifty years. In recent decades, a joint consideration of beamforming and cyclostationarity (i.e., beamforming for cyclostationary signals) attracted certain attention $[12,13]$. In those studies, the signals are both stationary and cyclostationary. In other words, continuous signals with repetitive structures are considered. In our work, we study noncontinuous signals with repetitive structures. Therefore, this paper exploits cyclostationarity to counter the nonstationarity problem in optimal beamforming.

The rest of the paper is organized as follows: Section 2 introduces the fundamentals of the WLPS structure; Section 3 discusses the implementation of WLPS system and the nonstationarity problem; Section 4 demonstrates how to exploit cyclostationarity to counter the nonstationarity problem; Section 5 presents numerical results, and Section 6 concludes the paper.

\section{WLPS BASIC STRUCTURE}

The WLPS comprises of a set of DBS and transponders. In the scope of this paper, we consider the communication between one DBS and multiple transponders. The DBS transmits ID request signals periodically to all transponders in

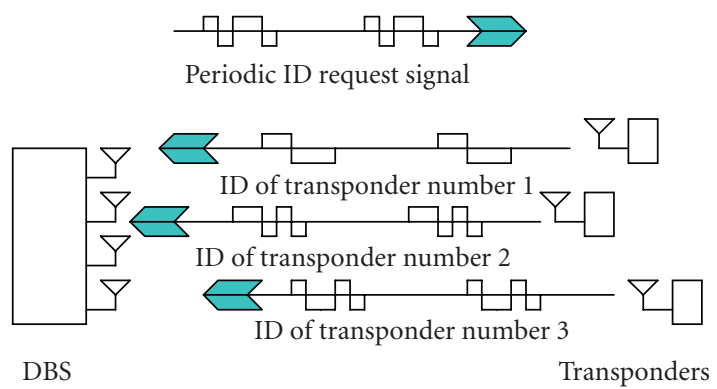

FIGURE 1: WLPS basic structure.

its coverage area. Once a transponder detects the ID request signal, it sends its unique ID (a signal with limited duration) back to the DBS, as shown in Figure 1. The DBS is equipped with multiple antennas to support DOA estimation and beamforming.

In the WLPS, a DBS communicates with multiple transponders simultaneously. This is the same as standard cellular communication systems. However, different from cellular systems, the DBS received signal in the WLPS is not stationary.

As shown in Figure 1, the signal transmitted by a transponders do not span over the whole time domain. This feature leads to a new performance measure metric: probabilityof-overlapping, $p_{\text {ovl }}$, which is defined as the probability that the desired ID is overlapped with the ID signals from other transponders. In standard wireless systems, $p_{\text {ovl }}$ is always unity for multiple transponders. In the DBS receiver, the probability of overlapping is less than unity and corresponds to:

$$
p_{\text {ovl }}=1-\left(1-d_{c}\right)^{K-1}
$$

where $K$ denotes the number of transponders and $d_{c}$ represents duty cycle, which is defined as:

$$
d_{c}=\frac{\tau}{\text { IRT }_{\min }}
$$

Here, $\tau$ is the duration of the ID of a transponder, and IRT min $_{\text {m }}$ is the time difference between the first responding transponder and the last responding transponder. A comprehensive results for $\mathrm{IRT}_{\min }$ have been introduced in [1]; here, roughly,

$$
\mathrm{IRT}_{\min }=\frac{R_{\max }}{2 c}
$$

where $R_{\max }$ is the maximum coverage distance of the DBS, and $c$ denotes the speed of light. For vehicle collision avoidance applications, typically $R_{\max }$ should not exceed $1 \mathrm{~km}$. The exact value of $R_{\max }$ may vary with different environments, for example, urban or highways.

In general, through this preliminary study, the noncontinuous nature of the WLPS seems alleviate the interference problem: the undesired signals from other transponders may or may not interfere with the desired signal. In contrast, in standard communication systems, the undesired signals always overlap with the desired signal. 


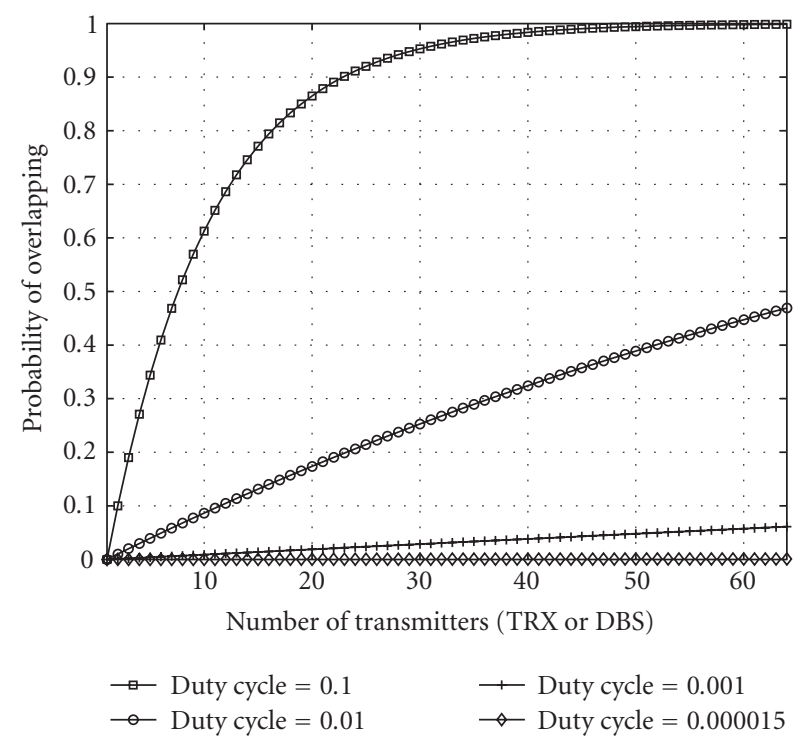

Figure 2: The probability of overlapping.

However, it is noted that the noncontinuous nature of the WLPS is not sufficient in terms of rejecting interference. As shown in Figure 2, the probability of overlapping is very high when $d_{c}=0.1$ with a moderate number of transponders $(N=10)$. In many applications, for example, vehicle collision avoidance, the duty cycle might be even larger than 0.1 . Therefore, one cannot expect to suppress interference reliably through the noncontinuous nature of the WLPS.

To reduce interference power, DS-CDMA and beamforming techniques are necessary in the WLPS. A detailed analysis for conventional beamforming and DS-CDMA techniques has been presented in [7]. In general, optimal beamformers perform better than the conventional beamformer. Hence, it is natural to extend our study from conventional beamformer to optimal beamformers.

Optimal beamformers generate a statistically optimum estimation of the desired signal through applying a weight vector to the observed data. This weight vector is computed via optimizing a certain cost function. Examples of these cost functions include total power, SINR, entropy, mean square error, or nonGaussianity [14, 15]. Here, LCMV beamformer is selected because: (1) it is particularly good at rejecting interference and (2) it only requires the observations of the received signals and the direction of the desired signal. The former is easy to obtain and the latter has been available via the DOA estimation process, which is prior to the beamforming process.

The basic structure of the WLPS has been introduced in this section. In the next section, we introduce the signal model of the WLPS and describe the beamforming implementation in a mathematical form. It is emphasized that directly applying LCMV beamforming in the WLPS is not appropriate due to its nonstationary nature. In Section 4, cyclostationarity would be exploited to solve the nonstationarity problem.

\section{SYSTEM IMPLEMENTATION AND NONSTATIONARITY ANALYSIS}

Once a transponder detects the ID request signal, it would transmit its unique ID back to the DBS. To suppress interference from other transponders, the bits in the ID are spread by DS-CDMA techniques. Hence, the transponders would periodically transmit DS-CDMA signals that are with a limited duration. In a multipath (urban) environments, the received signal at the DBS receiver would be the summation of DSCDMA signals from multiple transponders through multiple paths. Finally, in the DBS receiver, it is possible to apply DSCDMA despreading and beamforming techniques to extract the ID of the desired transponder, as explained in Section 3.1.

In this work, the DOA estimation for the paths of the desired transponder is assumed to be perfect. Although the nonstationarity nature does have effect on DOA estimation, the effect turns out to be minimal, and the DOA estimation is accurate enough for most practical applications [5]. Since the only required information for LCMV beamforming is the directions of the paths of the desired transponder and the estimation of covariance matrix, a good estimation of the covariance matrix would ensure a good ID detection performance, as depicted in Section 3.2.

In Section 3.3, it is shown that the standard sample covariance matrix estimator does not lead to a good quality of covariance matrix estimation. The reason is that due to the nonstationary nature of the WLPS, different bits of the ID experience difference interference. Hence, averaging covariance matrix over each bit does not lead to a consistent estimator, that is, increasing the number of averaged data does not reduce the mean square error (MSE) of the estimation. The consistent covariance matrix estimator, which exploits the cyclostationarity of the WLPS, would be introduced in Section 4.

\subsection{Signal model}

The transmitted DS-CDMA signal by the $k$ th transponder corresponds to

$$
\begin{aligned}
s^{k}(t)= & g_{\tau}(t) \\
& \cdot \sum_{n=0}^{N-1} b^{k}[n] \cdot g_{T_{b}}\left(t-n T_{b}\right) \cdot a^{k}\left(t-n T_{b}\right) \cdot \cos \left(2 \pi f_{c} t\right),
\end{aligned}
$$

where $N$ denotes the number of bits per ID code (that represents the maximum capacity of the WLPS, which is in the order of $\left.2^{N}\right), b^{k}[n]$ denotes the $n$th bit of transponder $k$ 's ID, $T_{b}=\tau / N$ represents the transponder bit duration, $g_{\tau}(t)$, and $g_{T_{b}}(t)$ are rectangular pulses with the duration of $\tau$ and $T_{b}$, respectively. Here, $a^{k}(t)$ denotes the spreading code for the $k$ th transponder, that is,

$$
a^{k}(t)=\sum_{g=0}^{G-1} C_{g}^{k} g_{T_{c}}\left(t-g T_{b}\right), \quad C_{g}^{k} \in\{-1,1\}
$$


where $G\left(G \leq 2^{N}\right)^{1}$ is the processing gain (code length), $T_{c}=$ $T_{b} / G=\tau /(N \cdot G)$ represents the chip duration, and $g_{T_{c}}(t)$ is a rectangular pulse with the duration of $T_{c}$.

With an antenna array mounted on the DBS receiver, the received signal at the DBS (see Figure 3), which is the summation of signals from multiple transponders through multiple paths, corresponds to

$$
\begin{array}{r}
\vec{r}(t)=\sum_{k=1}^{K} \sum_{l=0}^{L^{k}-1} \sum_{n=0}^{N-1} \alpha_{l}^{k} \vec{V}\left(\theta_{l}^{k}\right) b^{k}[n] g_{T_{b}}\left(t-\tau_{l}^{k}-n T_{b}\right) g_{\tau}\left(t-\tau_{l}^{k}\right) \\
\cdot a^{k}\left(t-\tau_{l}^{k}-n T_{b}\right) \cos \left(2 \pi f_{c} t+\phi_{l}^{k}\right)+\vec{n}(t),
\end{array}
$$

where $K$ denotes the total number of transponders, $L^{k}$ is the number of paths for the transponder $k$, and $\alpha_{l}^{k}, \tau_{l}^{k}, \phi_{l}^{k}$ denote the fading factor, time delay, and random phase shift for $k$ th transponder's $l$ th path, respectively. Here, for simplicity of presentation, we assume that $L^{k}=L$, for all $k \cdot \vec{V}\left(\theta_{l}^{k}\right)$ denotes the array response vector that corresponds to

$$
\begin{aligned}
\vec{V}\left(\theta_{l}^{k}\right)= & {\left[1 \exp \left(-i \cdot 2 \pi d \cos \left(\theta_{l}^{k}\right) / \lambda\right) \cdots\right.} \\
& \left.\exp \left(-i \cdot 2(M-1) \pi d \cos \left(\theta_{l}^{k}\right) / \lambda\right)\right]^{T} .
\end{aligned}
$$

Here, $i$ denotes the imaginary unit, $d$ is the spacing between antenna elements, $M$ is the total number of antennas, $(\cdot)^{T}$ denotes transpose, $\lambda$ denotes the carrier wavelength, and $\theta_{l}^{k}$ is the direction of $k$ th transponder's $l$ th path. Basically, in (7), we assume half wavelength spacing between antennas and the precise knowledge of array manifold at the DBS receiver.

After demodulation, the $g$ th chip of the $n$th bit output for the $j$ th transponder's, the $q$ th path would correspond to

$$
\begin{aligned}
\vec{y}_{q}^{j}[n, g]= & \int_{\tau_{q}^{j}+n T_{b}+g T_{c}}^{\tau_{q}^{j}+(n+1) T_{b}+(g+1) T_{c}} \vec{r}(t) \\
& \times \cos \left(2 \pi f_{c} t+\phi_{q}^{j}\right) g\left(t-\tau_{q}^{j}-n T_{b}-g T_{c}\right) d t .
\end{aligned}
$$

The $g$ th chip of the $n$th bit output of the beamformer for $j$ th transponder's $q$ th path is given as

$$
z_{q}^{j}[n, g]=\vec{W}^{H}\left(\theta_{q}^{j}\right) \cdot \vec{y}_{q}^{j}[n, g],
$$

where the weight vector $\vec{W}\left(\theta_{q}^{j}\right)$ and $\vec{y}_{q}^{j}[n, q]$ are both $1 \times M$ column vectors, and $H$ denotes Hermitian transpose.

The receiver in Figure 3 and (9) resembles a spatial RAKE-like structure. Here, each RAKE corresponds to one path. Each path is received from a specific direction. Hence, beamforming on each RAKE is applied to capture the energy from the associated direction.

After beamforming, the signals from different paths are combined via maximal Ratio combining:

$$
z^{j}[n, g]=\sum_{l=1}^{L} \alpha_{l}^{j} z_{l}^{j}[n, g] .
$$

\footnotetext{
${ }^{1}$ Note that $2^{N}$ is the maximum number of transponders that the system can accommodate.
}

Finally, the CDMA despreading is applied and the detected bit is given as

$$
z^{j}[n]=\sum_{g=1}^{G} z^{j}[n, g] C_{g}^{j} .
$$

The above description has included all necessary steps of WLPS ID detection process, except the calculation of the weight vector $\vec{W}\left(\theta_{q}^{j}\right)$ in (9), which is the kernel part of this work. Here, we discuss how to determine $\vec{W}\left(\theta_{q}^{j}\right)$ in Section 3.2.

\subsection{Weight vector calculation}

The conventional beamforming weight vector simply corresponds to

$$
\vec{W}_{f}\left(\theta_{q}^{j}\right)=\vec{V}\left(\theta_{q}^{j}\right)
$$

Noting that $\vec{V}\left(\theta_{q}^{j}\right)$ is a predefined linear phase filter, which coincides with the definition of discrete Fourier transform, it is said that the conventional beamforming is equivalent to discrete Fourier transform [16].

The LCMV beamforming, which minimizes the total output power, while keeping the desired signal power constant, corresponds to the solution of the following optimization problem $[8]$ :

$$
\min _{\vec{W}_{c}\left(\theta_{q}^{j}\right)} \vec{W}_{c}^{H}\left(\theta_{q}^{j}\right) \mathbf{R}_{q}^{j} \vec{W}_{c}\left(\theta_{q}^{j}\right) \quad \text { s.t. } \vec{W}_{c}^{H}\left(\theta_{q}^{j}\right) \vec{V}\left(\theta_{q}^{j}\right)=1 .
$$

Using Lagrange multiplier, the solution of the above equation, that is, LCMV BF, is given by [17]:

$$
\vec{W}_{c}\left(\theta_{q}^{j}\right)=\frac{\mathbf{R}_{q}^{j^{-1}} \vec{W}_{f}\left(\theta_{q}^{j}\right)}{\vec{W}_{f}^{H}\left(\theta_{q}^{j}\right) \mathbf{R}_{q}^{j^{-1}} \vec{W}_{f}\left(\theta_{q}^{j}\right)},
$$

where $\mathbf{R}_{q}^{j}$ is the covariance matrix of $j$ th transponder's $q$ th path's observed signal, that is, $\mathbf{R}_{q}^{j}=E\left[\vec{y}_{q}^{j} \cdot \vec{y}_{q}^{j^{H}}\right]$.

In this work, precise knowledge of the DOA $\theta_{q}^{j}$ and array manifold is assumed, that is, $\vec{W}_{f}\left(\theta_{q}^{j}\right)$ is perfectly known. Then, the only left important implementation issue of the LCMV beamforming is the estimation of $\mathbf{R}_{q}^{j}$. In general, the sample covariance matrix estimator corresponds to

$$
\widehat{\mathbf{R}}_{q}^{j}=\frac{1}{\Gamma} \sum_{n=0}^{\Gamma-1} \vec{y}_{q}^{j}[n] \vec{y}_{q}^{j^{H}}[n],
$$

where $\Gamma,(\Gamma \in\{1,2,3 \cdots N\})$, denotes the selected data length for $\mathbf{R}_{q}^{j}$ estimation. If $\vec{y}_{q}^{j}[n]$ is a stationary and ergodic process, the sample average equals time average, and the sample covariance matrix estimator leads to an accurate estimate of $\mathbf{R}_{q}^{j}$. In another word, the sample covariance matrix estimator would be consistent, and increasing the number of data samples reduces the error variance of the sample covariance matrix estimator. 


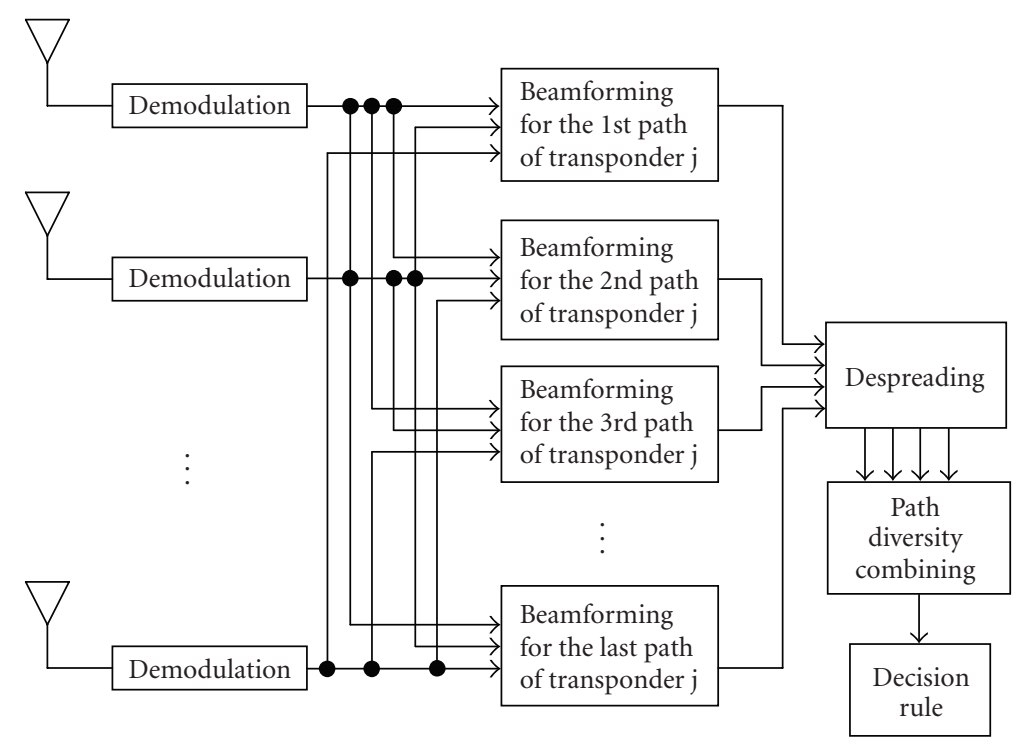

FIgURE 3: DBS receiver implementation via antenna arrays and DS-CDMA systems.

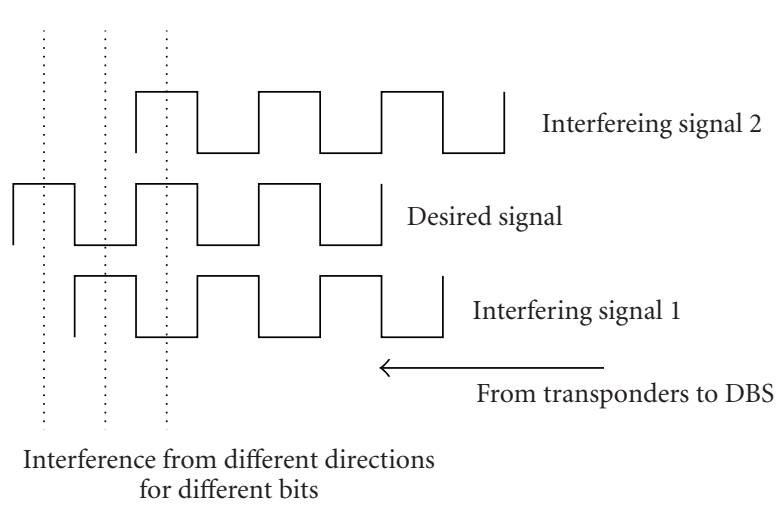

Figure 4: Different chips experience different interference.

\subsection{Nonstationarity analysis}

Standard wireless communication systems are stationary because of transmission of very long sequences from a large number of users. In other words, in these systems, different chips of the desired signal would experience the same interference. However, because the WLPS transponder transmitted signal is a short burst signal, the interfering signal may only interfere with some, but not all chips of the desired signal (see Figure 4). Hence, the interference changes within each bit of the desired signal. This is especially the case for medium probability-of-overlapping, $p_{\text {ovl }}$, values. Therefore, in WLPS, $\mathbf{R}_{q}^{j}$ varies for different chips and large selection of $\Gamma$ does not necessarily lead to a high quality of the covariance matrix estimation. To have a better understanding when the received signal is not stationary, we have the following discussion.

(i) Small values of $d_{c}$ in (1) leads to low $p_{\text {ovl }}$ (see Figure 2). In an extreme situation, $p_{\text {ovl }} \rightarrow 0$. In this case, since there is no interference at all, $E\left[\vec{y}_{q}^{j}[n] \vec{y}_{q}^{H}[n]\right]=$ $E\left[\vec{y}_{q}^{j}[n+1] \vec{y}_{q}^{j^{H}}[n+1]\right]$ and the sample covariance matrix estimator leads to an accurate estimation. However, the main advantage of LCMV beamforming is interference suppression, and in this situation, LCMV will not provide better performance than conventional beamforming even with accurate estimation of $\mathbf{R}_{q}^{j}$.

(ii) Large values of $d_{c}$ in dense transponder environment leads to $p_{\text {ovl }} \rightarrow 1$. In this case, the sum of interferences would approximately be white noise, and the received signal statistically tends to be stationary, that is, $E\left[\vec{y}_{q}^{j}[n] \vec{y}_{q}^{j^{H}}[n]\right] \simeq E\left[\vec{y}_{q}^{j}[n+1] \vec{y}_{q}^{j^{H}}[n+1]\right]$. In this case, the covariance matrix would be an identity matrix and LCMV beamforming becomes equivalent to conventional beamforming.

(iii) Medium $d_{c}$ values and moderate transponder density lead to a spatial structure for the interference, that is, several interfering signals are received in different directions. In this case, the received data samples would be nonstationary, large selection of $\Gamma$ does not improve the quality of covariance estimation, and the sample covariance matrix estimator is not consistent.

Figure 5 represents the mean square error (MSE) between the true value and the estimated values of covariance matrix as a measure of nonstationarity, assuming a flat fading channel. The MSE corresponds to

$$
\operatorname{MSE}=\sum_{m=1}^{M} \sum_{u=1}^{M}\left(\mathbf{R}_{q}^{j}(m, u)-\hat{\mathbf{R}}_{q}^{j}(m, u)\right)^{2},
$$

where $M$ is the number of antenna array elements, $\mathbf{R}_{q}^{j}$ and $\widehat{\mathbf{R}}_{q}^{j}$ denote the true and estimated covariance matrixes via sample covariance matrix, respectively. The direction and distance of the transponders are assumed to be uniformly distributed 


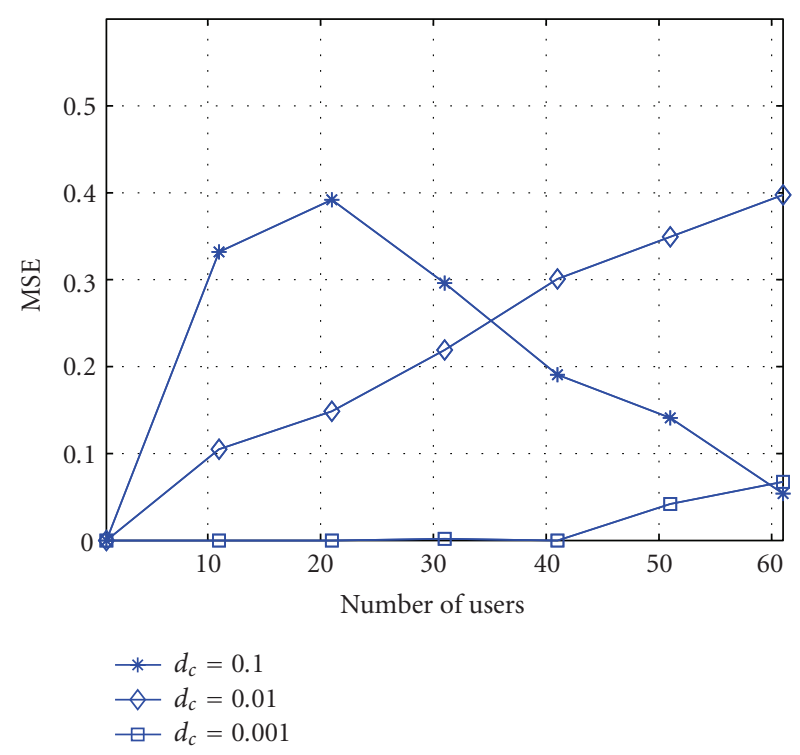

FIGURE 5: Simulation results: the mean square error of estimated covariance matrix by standard estimation method.

in $[0, \pi]$ and $\left[0, R_{\max }\right]$. The estimated covariance matrix is normalized before comparing it with true covariance matrix.

It is seen that when $d_{c}$ is small $(=0.001)$ and the number of transponder is small $(<30)$, the MSE is kept minimal, which is consistent with the first case discussed above.

When $d_{c}$ is large $(=0.1)$ and the number of transponder is large ( $>60)$, the MSE is small as well. This corresponds to the second case discussed. A large number of interferences lead to a spatially white structure. In other words, every chip is interfered by signals in many directions. Hence, the interference over different chips would be similar, which leads to a stationary process.

When $d_{c}$ is moderate $(=0.01)$, the MSE is large, that is, the nonstationarity problem is severe.

The high MSE shown in Figure 5 leads to low probability of detection. As a result, directly applying LCMV beamforming does not improve the system performance compared to a conventional beamforming. This point is verified by ID detection simulations in Figure 11 (see Section 5).

\section{ESTIMATOR BASED ON THE CYCLOSTATIONARITY}

Section 3.3 introduced the nonstationarity problem in the WLPS. This section proposes a modified covariance matrix estimator to solve the nonstationarity problem, which exploits the cyclostationarity property of the WLPS.

\subsection{New estimator via cyclostationarity}

The nonstationarity is mainly generated by the noncontinuous transmission of transponders. However, it should be noted that, in addition to the noncontinuousness, the transmission is also periodical. In every period, a transponder retransmits the same ID bits with the same spreading code. Now, assuming all transponders' directions and distances remain the same for a number of periods, same chips of transponder ID in different period experience the same interference (See Figure 6). Here, the period of transponder transmission is called ID request time (IRT).

The repetition property of transponder transmission is also known as cyclostationarity: although different chips in the same period does not experience same interferences, same chips in different periods experience same interferences. Hence, it is possible to apply beamforming to each chip, if the covariance matrix for each chip can be estimated. As shown in Figure 6, the covariance matrix estimation via cyclostationarity for the $g$ th chip of the $n$th bit corresponds to

$$
\hat{\mathbf{R}}_{q}^{j}[n, g]=\frac{1}{\Omega} \sum_{\omega=1}^{\Omega} \vec{y}_{q}^{j}[n, g, \omega] \vec{y}_{q}^{j^{H}}[n, g, \omega]
$$

where $\Omega$ denotes the number of period within which the cyclostationarity holds. Using (17), consequently (8) and (9) would correspond to

$$
\begin{aligned}
& \vec{y}_{q}^{j}[n, g, \omega] \\
& =\int_{\tau_{q}^{j}+n T_{b}+g T_{c}+(\omega-1) \mathrm{IRT}}^{\tau_{q}^{j}+(n+1) T_{b}+(g+1) T_{c}+(\omega-1) \mathrm{IRT}} \vec{r}(t) \cos \left(2 \pi f_{c} t+\phi_{q}^{j}\right) \\
& \quad \cdot g\left(t-\tau_{q}^{j}-n T_{b}-g T_{c}-\omega \mathrm{IRT}\right) d t, \quad \omega \in\{1,2, \ldots, \Omega\}, \\
& z_{q}^{j}[n, g]=\frac{1}{\Omega} \sum_{\omega=1}^{\Omega} \vec{W}^{H}\left(\theta_{q}^{j}\right) \cdot \vec{y}_{q}^{j}[n, g, \omega],
\end{aligned}
$$

respectively. Equation (19) reflects both beamforming and equal gain time diversity combining processes. Because each frame experiences independent fading, we also achieve time diversity benefits via combining the chips from different IRT. The receiver structure via cyclostationarity is shown in Figure 7. Here, a separate block is considered for the covariance matrix estimator via cyclostationarity, since the new estimator requires a temporary storage of the received signals.

It should be noted that the proposed consistent covariance matrix estimator may not be restricted to LCMV beamforming, various optimal [18] or robust beamforming $[19,20]$ methods may also use this estimator. In this paper, the application of LCMV beamforming in the WLPS is introduced. The proposed concept may be easily extended to any signal processing algorithm that requires an estimation of covariance matrix, as long as the system exhibits a repetitive nature.

\subsection{Cyclostationarity duration}

An important issue of the new estimator is the maximum possible value of $\Omega$, that is, the number of periods that the cyclostationarity holds. A larger value of $\Omega$ leads to better estimation, while a small value of $\Omega$ (e.g., 1 or 2) will render the estimator via cyclostationarity improper. 


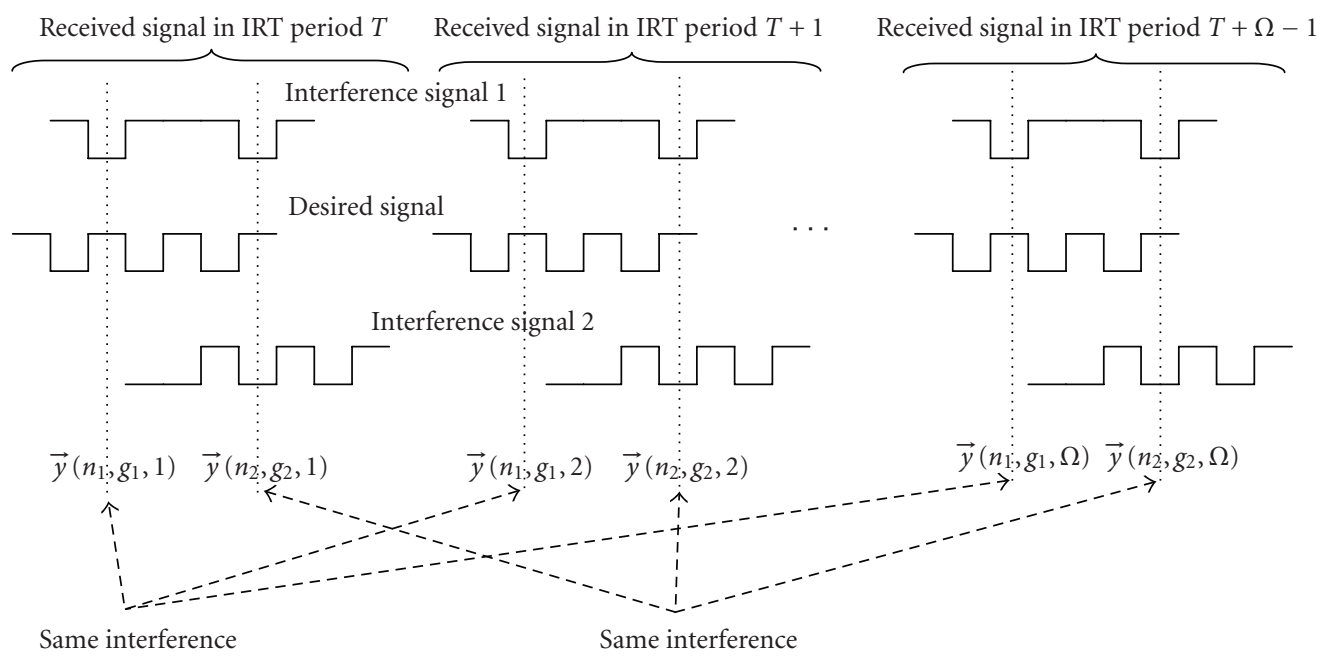

FIGURE 6: Same chips in different IRT periods have the same interference.

\subsubsection{Cyclostationarity duration for a single transponder}

Basically, $\Omega$ is determined by IRT and the duration within which the cyclostationarity remains available, and corresponds to

$$
\Omega \leq \frac{T_{c y}}{\text { IRT }}
$$

where $T_{c y}$ is the time within which cyclostationarity condition holds, and IRT denotes the repetition time of the ID request signal. Two parameters impact the cyclostationarity: The direction and the distance of transponder. Hence, the $T_{c y}$ is the time within which (a) the direction of the transponder approximately remains constant and (b) the distance of the transponder approximately remains unchanged (see Figure 8).

Therefore, we consider the impact of the movement of the transponder in two directions. The first is in the direction that is parallel to the line connecting transponder and antenna array. In this direction, the variation of the TOA within the duration of $T_{c y}$ should be much smaller than the chip duration $T_{c h}$, that is, TOA is relatively fixed during $T_{c y}$, which corresponds to

$$
T_{c y} \ll \frac{c}{B \cdot v_{\|}},
$$

where $c$ is the speed of light, $B=1 / T_{c h}$ denotes the transponder signal bandwidth, and $v_{\|}$represents the Doppler velocity of the transponder;

The second direction is the direction that is perpendicular to the line connecting transponder and antenna array. In this direction, the variation of DOA should be much smaller than the antenna array half power beamwidth, that is, DOA is relatively fixed during $T_{c y}$, which corresponds to

$$
T_{c y} \ll \frac{\left(\theta_{B} / 2\right) \cdot d}{v_{\perp}},
$$

where $\theta_{B}$ is the half power beam width, $d$ denotes the distance between transponder and DBS, and $v_{\perp}$ is depicted in Figure 8.

Combining the above two conditions, the final condition corresponds to

$$
T_{c y} \ll \min \left(\frac{c}{B \cdot v_{\|}}, \frac{\theta_{B} \cdot d}{2 v_{\perp}}\right) .
$$

Note that the first condition (TOA constraint) is independent of distance, while the second condition (DOA constraint) depends on both velocity and distance.

Equivalent to (23), we have the conditions for cyclostationarity Doppler frequency, which corresponds to

$$
f_{c y}=\frac{1}{T_{c y}} \gg \max \left(\frac{B \cdot v_{\|}}{c}, \frac{2 v_{\perp}}{\theta_{B} \cdot d}\right) .
$$

This means that the changing rate of cyclostationarity should be much larger than DOA/TOA changing rate.

Knowing $v_{\|}=v \cdot \cos (\psi)$ and $v_{\perp}=v \cdot \sin (\psi)$ (see Figure 8) and considering $\psi$ a uniform random variable within 0 and $2 \pi$, the cyclostationarity Doppler spread $\left(B_{c y, d}\right)$, which is the root-mean-square (RMS) value of cyclostationarity Doppler frequency, corresponds to

$$
B_{c y, d}=\max \left(\sqrt{Æ\left[\frac{B \cdot v_{\|}}{c}\right]^{2}}, \sqrt{Æ\left[\frac{2 v_{\perp}}{B_{d} \cdot d}\right]^{2}}\right),
$$

where $Æ(\cdot)$ denotes expectation operation.

Applying simple mathematical manipulations (25) would correspond to

$$
B_{c y, d}=\max \left(\frac{B \cdot v}{\sqrt{2} c}, \frac{\sqrt{2} v}{\theta_{B} \cdot d}\right) .
$$

Then, using (26) and similar to the definition of channel coherence time, we define the cyclostationarity coherence time as [21]

$$
T_{c y, c} \cong \frac{1}{B_{c y, d}} .
$$




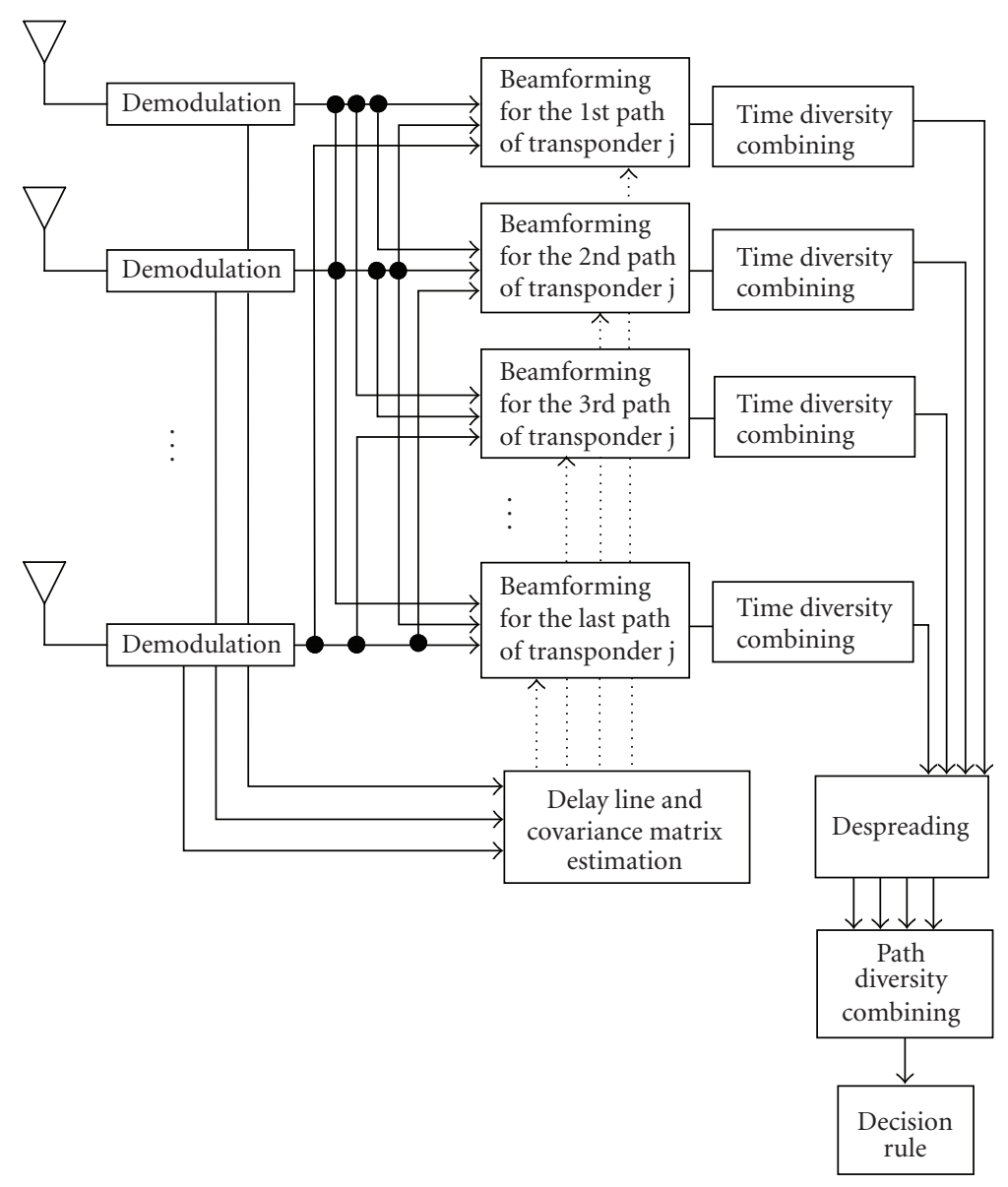

FIGURE 7: Receiver structure with using cyclostationarity.

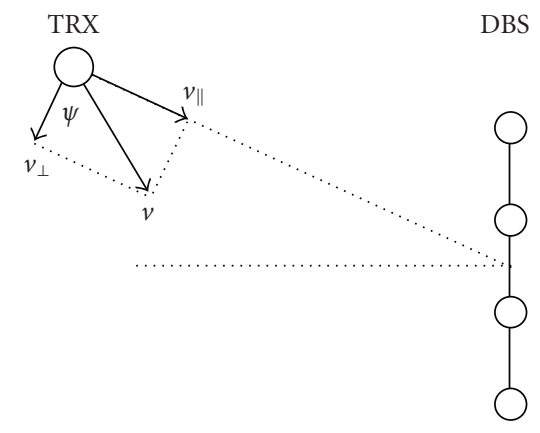

Figure 8: Relationship between $v, v_{\perp}$, and $v_{\|}$.

In order to guarantee cyclostationarity during $T_{c y}, T_{c y}$ should be selected smaller than $T_{c y, c}$, or

$$
T_{c y}<T_{c y, c}
$$

To demonstrate the effects of the two conditions on cyclostationarity, the cyclostationarity coherence time with various velocity and distance values has been computed in Figure 9. Here, we assume $300 \mathrm{MHz}$ bandwidth and $27^{\circ}$ half power beamwidth (consistent with four antenna elements). The first area of interest in Figure 9 is low-velocity and shortrange area, which is mainly suitable for applications such as indoor and airport security. Note that the cyclostationarity Doppler spread varies with distance in this area. Hence, we can conclude that for short range applications, DOA would be the dominant condition for cyclostationarity. The second area of interest is high-velocity, long-range area, which is mainly suitable for vehicle collision avoidance system. Note that the cyclostationarity Doppler spread is independent of distance in this area. We can conclude that for long-range applications, the main constraint is the rate of change of TOA.

\subsubsection{Cyclostationarity duration for multiple transponders}

The cyclostationarity duration for a single transponder is straightforward. However, in the WLPS system, multiple transponders may present. In this situation, the cyclostationarity duration computation is much more complicated. Here, we compute the probability $\left(P_{c s}\right)$ that the position of 
all transponders remain relatively fixed in $\left(T_{c s}\right)$ seconds, that is,

$$
\begin{aligned}
& P_{c s}=\operatorname{prob}\{ \text { The position of all transponder } \\
&\text { nodes remain unchanged within } \left.T_{c s}\right\} \leq p,
\end{aligned}
$$

where $p$ is generally selected close to unity. Assuming positioning statistics of different transponders are independent, then

$$
P_{c s}=\prod_{m=1}^{M} \gamma^{(m)}
$$

where $\gamma^{(m)}$ refers to the probability that the $m$ th transponder node remains unchanged during $T_{c s}$. Based on the discussions of Section 4.2.1, $\gamma^{(m)}$ corresponds to

$$
\gamma^{(m)}=\operatorname{prob}\left\{v_{\|}^{(m)} \ll \frac{c}{B \cdot T_{c s}}, \frac{v_{\perp}^{(m)}}{d^{(m)}} \ll \frac{\theta_{B}}{2 T_{c s}}\right\} .
$$

Now the same movement statistics is assumed for all transponders: (i) the speed of each transponder node, $v^{m}$ follows Rayleigh distribution with mean $m_{v}$; (ii) direction of each transponder node, $\psi^{(m)}$, is uniformly distributed in $[0,2 \pi)$; and (iii) all transponder nodes are uniformly distributed in DBS coverage area, that is, $R^{(m)}$ is uniform in $\left(0, R_{\max }\right.$ ), where $R_{\max }$ is the maximum radius of the DBS coverage.

Using assumptions (i) and (ii), $v_{\|}^{(m)}=v^{(m)} \sin \psi^{(m)}$ and $v_{\perp}^{(m)}=v^{(m)} \cos \psi^{(m)}$ would be two independent random variables with zero mean and variance $\sigma=\sqrt{2 / \pi} m_{v}$ for all $m \in\{1,2, \ldots, M\}$ transponders. Let $X^{(m)}=v_{\|}^{(m)}$ and $Y^{(m)}=v_{\perp}^{(m)} / d^{(m)}$, then (31) would correspond to

$$
\gamma^{(m)}=\operatorname{prob}\left\{X^{(m)}<\alpha, Y^{(m)}<\beta\right\}
$$

where $\alpha=(1 / \Omega)\left(c / B \cdot T_{c s}\right), \beta=(1 / \Omega)\left(\theta_{B} / 2 T_{c s}\right), B$ is introduced in (21), and $\Omega$ is a constant that satisfies $\Omega \gg 1$.

Note that $X^{(m)}$ and $Y^{(m)}$ are two independent random variables; hence,

$$
\operatorname{prob}\left\{X^{(m)}<\alpha, Y^{(m)}<\beta\right\}=F_{X}^{(m)}(\alpha) \cdot F_{Y}^{(m)}(\beta)
$$

$F_{X}^{(m)}(\alpha)$ and $F_{Y}^{(m)}(\beta)$ are cumulative distribution functions $(\mathrm{CDF})$ of $X^{(m)}$ and $Y^{(m)}$, respectively, that is,

$$
\begin{gathered}
F_{X}^{(m)}(\alpha)=\frac{1}{2}+\frac{1}{2} \operatorname{erf}\left(\frac{\alpha}{\sqrt{2} \sigma}\right) \text { for any } m \\
F_{Y}^{(m)}=\frac{1}{2}+\frac{1}{2 R_{\min }}\left(R_{\max } \operatorname{erf}\left(\frac{R_{\max } \beta}{\sqrt{2} \sigma}\right)\right. \\
\left.+\sqrt{\frac{2}{\pi}} \frac{\sigma}{\beta}\left(1-e^{-R_{\max }^{2} \beta^{2} / \sqrt{2} \sigma^{2}}\right)\right),
\end{gathered}
$$

where $\operatorname{erf}(x)=(2 / \sqrt{\pi}) \int_{0}^{x} e^{-t^{2}} d t$.

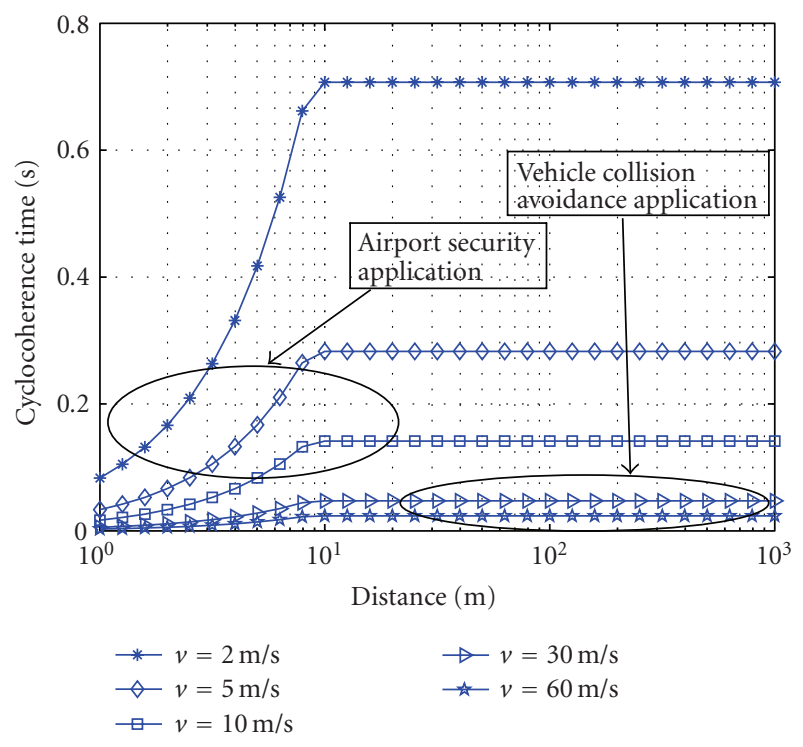

FIGURE 9: Cyclostationarity coherence time for different applications, single transponder.

Assuming all transponders have the same movement statistics, we would have $\gamma^{(m)}=\gamma$ and $P_{c s}=\gamma^{M}$. Incorporating (32), (33), and (34), $P_{c s}$ in (29) would correspond to

$$
\begin{aligned}
P_{c s}= & \left(\frac{1}{2}+\frac{1}{2} \operatorname{erf}\left(\frac{\alpha}{\sqrt{2} \sigma}\right)\right)^{M} \\
& \cdot\left(\frac{1}{2}+\frac{1}{2 R_{\min }}\left(R_{\max } \operatorname{erf}\left(\frac{R_{\max } \beta}{\sqrt{2} \sigma}\right)\right.\right. \\
& \left.\left.\quad+\sqrt{\frac{2}{\pi}} \frac{\sigma}{\beta}\left(1-e^{-R_{\max }^{2} \beta^{2} / \sqrt{2} \sigma^{2}}\right)\right)\right)^{M} .
\end{aligned}
$$

Note that $\beta=s \alpha\left(s=B \cdot \theta_{B} / 2 c\right)$, then (35) would be a fixedpoint equation of $\alpha$. Based on the definition of $\alpha$ in (32)

$$
T_{c s}=\frac{1}{\Omega} \frac{c}{B \cdot \alpha} \text {. }
$$

Hence, $\alpha$ is a function of the DBS antenna array half power beamwidth and coverage range, the number of transponders, transponder speed, and transponder pulse duration. As a result, $T_{c s}$ would be a function of those parameters.

Solving (35) and finding an analytic solution for $T_{c s}$ is not trivial. Hence, in Figure 10, numerical results for $T_{c s}$ are generated in terms of (a) the number of transponder and transponder average speed for a system with (uniform linear array with 4 elements and half wavelength element spacing) and (transponder bandwidth of $8.33 \mathrm{MHz}$ ) and (b) transponder bandwidth and DBS antenna array half power beamwidth for a system with $M=10$ transponders with average speed of $m_{v}=5 \mathrm{~m} / \mathrm{s}$. In these simulations, other selected parameters are $R_{\max }=1000 \mathrm{~m}, p=0.95$ [see (29)], and $\Omega=10$. It is observed that $T_{c s}$ decreases as the number of transponders, transponder average speed, and bandwidth increase. Moreover, $T_{c s}$ decreases as half power beamwidth of 


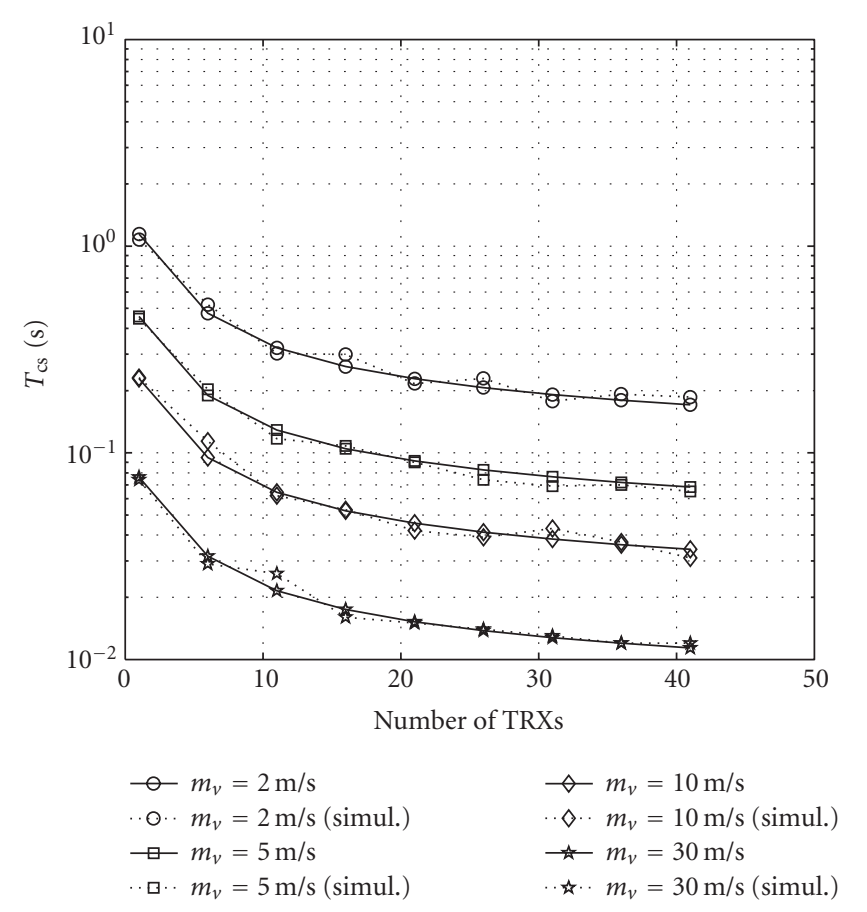

FIGURE 10: Cyclostationarity coherence time for multiple transponders.

the antenna array decreases (e.g., using more elements in the array). The doted curves in Figure 10 represents the simulation results generated using similar assumptions. The theoretical results have a good match with numerical results.

The standard sample covariance estimator does not perform for nonstationary signals. Hence, its MSE does not alter with the number of temporal samples. In contrast, the proposed cyclostationary-based covariance matrix estimator improves the MSE as the number of samples increases. The number of samples increases as the cyclostationary duration increases. In Section 4, we substantially discussed that the cyclostationarity duration is sufficiently long in practical situations. Hence, the MSE for the proposed estimator is small enough for most practical applications. Numerical results in Section 5 verify this claim.

\section{NUMERICAL RESULTS}

In this section, we use MonteCarlo simulations to evaluate the ID detection performance of the WLPS system implemented via LCMV beamforming with and without the newly proposed covariance matrix estimator via the cyclostationarity property. Here, we consider a multitransponder, multipath environment. For simulation purposes, we assume the following:

(1) the ID code has 6 bits $(N=6)$;

(2) the DS-CDMA code has 64 chips $(G=64)$;

(3) channel delay spread for a typical street area is 27 nanoseconds [22];
(4) carrier frequency $=3 \mathrm{GHz}, \tau_{\mathrm{TRX}}=1.2 \mu \mathrm{s}$, and $\tau_{\mathrm{DBS}}=$ $24 \mu \mathrm{s}$

(5) the antenna array is linear with 4 elements, and element spacing $d=\lambda / 2=0.05 \mathrm{~m}$ (half power beamwidth $=27^{\circ}$ );

(6) four multipaths lead to $L=4$ fold path diversity;

(7) the transponder distance and angle are uniformly distributed in [ $\left[\begin{array}{ll}0 & 1\end{array}\right] \mathrm{km}$ and $\left[\begin{array}{ll}0 & \pi\end{array}\right]$, respectively;

(8) uniform multipath intensity profile, that is, bit energy is distributed in each path identically;

(9) binary phase shift keying (BPSK) modulation;

(10) perfect power control and DOA/TOA estimation.

The above assumptions are particularly suitable for vehicle safety applications. Based on the assumed setup, transponder signal TOA is uniformly distributed in $\left[\begin{array}{ll}T_{d} & T_{\max }\end{array}\right]$ at the DBS receiver. Assuming that $T_{d} \ll T_{\max }$, approximately TOA of transponder signal is uniformly distributed in $\left[\begin{array}{ll}0 & T_{\max }\end{array}\right]$, and the required bandwidth of a DS-CDMA transponder transmitter is $320 \mathrm{MHz}$. Using these parameters, $\mathrm{IRT}_{\min }=12 \mu \mathrm{s}$, then the duty cycle for DBS receivers would correspond to $d_{c \text {,DBS }} \simeq 0.1$, which leads to a high probability of overlapping (see Figure 2 ).

Assuming that the vehicle speed is $30 \mathrm{~m} / \mathrm{s}$, the cyclostationarity coherence time (based on an average distance of $500 \mathrm{~m}$ ) would be 47.1 milliseconds, as shown in Figure 9. As we mentioned in Section 2, usually IRT is selected much larger than $\mathrm{IRT}_{\min }$ in order to reduce interference power at transponder receiver. Here, we select IRT $=1.2$ milliseconds. Using (28), $T_{c y} \cong T_{c y, c} / 5=9.42$ milliseconds, and using (20), finally $\Omega \cong 8$. In other words, within 8 IRT frames, the conditions for cyclostationarity would well exist. It should be mentioned that the conditions simulated in this paper lead to a conservative selection of $\Omega$, and in many applications, higher value than $\Omega=8$ is expected.

The simulation results are shown in Figure 11. The measurement of ID detection performance is probability of miss detection $\left(P_{\mathrm{md}}\right)$, that is, the probability that the ID of the desired transponder is not detected correctly. Here, $P_{\mathrm{md}}=$ $1-\left(1-P_{d}\right)^{N}$, where $N$ is the number of bits per ID and $P_{d}$ denotes the probability that one bit of the ID is detected correctly. As discussed in Section 3.3, due to nonstationarity nature of the WLPS, traditional sample covariance matrix estimator computation leads to a high probability of miss detection. It can also be seen that the performance of LCMV BF with the covariance matrix estimator via cyclostationary property leads to a significantly improved performance compared to the standard covariance matrix estimator. It is observed that the proposed technique doubles the capacity of this system at the $P_{\mathrm{md}}=10^{-3}$ (i.e., from 25 to 50 ).

The result not only benefits from solving nonstationarity problem, but also the time diversity attained over the 8 IRT periods, since the fading is assumed to be independent over chips in different frames (IRT). This diversity improves the performance in conjunction with cyclostationarity. In order to demonstrate the different effects of time diversity combining and optimum beamforming, we also perform the optimum beamforming without using time diversity combining. It can be seen that both of the two techniques contributes to 


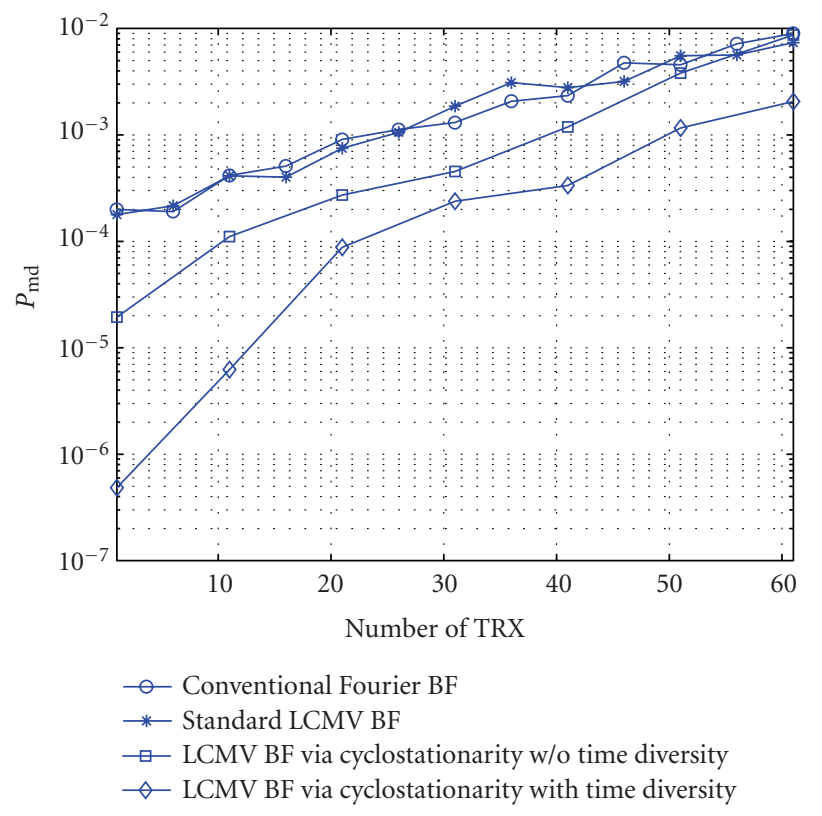

FIGURE 11: LCMV BF result with using cyclostationarity.

DBS receiver performance. It is observed that as the number of transponders increases, the time diversity has a dominant impact on the performance improvement.

\section{CONCLUSION}

This paper proposes a novel covariance matrix estimator, which is the critical step for optimal beamforming implementation, in a wireless local positioning system with a periodic signaling structure. Different from standard wireless systems, the standard sample covariance matrix estimator is not consistent in the WLPS system due to its nonstationarity nature. A new consistent estimator, which exploits the cyclostationarity property of the WLPS, is proposed. It is demonstrated that in most applications, the cyclostationarity duration is sufficiently large for covariance matrix estimation. Numerical simulations verify that the new estimator improves system performance significantly.

\section{ACKNOWLEDGMENTS}

This work was partially reported in [1]. This work is supported by the US NSF Grant ECS-0427430. WLPS US Patent is Pending at Michigan Tech. University.

\section{REFERENCES}

[1] H. Tong and S. A. Zekavat, "LCMV beamforming for a novel wireless local positioning system: a stationarity analysis," in Sensors, and Command, Control, Communications, and Intelligence (C3I) Technologies for Homeland Security and Homeland Defense IV, vol. 5778 of Proceedings of SPIE, pp. 851-862, Orlando, Fla, USA, March-April 2005.
[2] P. Stoica and A. Nehorai, "MUSIC, maximum likelihood, and Cramer-Rao bound," IEEE Transactions on Acoustics, Speech, and Signal Processing, vol. 37, no. 5, pp. 720-741, 1989.

[3] M. Hellebrandt, R. Mathar, and M. Scheibenbogen, "Estimating position and velocity of mobiles in a cellular radio network," IEEE Transactions on Vehicular Technology, vol. 46, no. 1, pp. 65-71, 1997.

[4] A. Juels, "RFID security and privacy: a research survey," IEEE Journal on Selected Areas in Communications, vol. 24, no. 2, pp. 381-394, 2006.

[5] J. Pourrostam, S. A. Zekavat, and H. Tong, "Novel directionof-arrival estimation techniques for periodic-sense local positioning systems," in Proceedings of the IEEE Radar Conference (RADAR '07), Waltham, Mass, USA, April 2007.

[6] Z. Wang and S. A. Zekavat, "Manet localization via multi-node TOA-DOA optimal fusion," in Proceedings of the Military Communications Conference (MILCOM '06), pp. 1-7, Washington, DC, USA, October 2006.

[7] H. Tong and S. A. Zekavat, "A novel wireless local positioning system via a merger of DS-CDMA and beamforming: probability-of-detection performance analysis under array perturbations," IEEE Transactions on Vehicular Technology, vol. 56, no. 3, pp. 1307-1320, 2007.

[8] O. L. Frost, "An algorithm for linearly constrained adaptive array processing," Proceedings of the IEEE, vol. 60, no. 8, pp. 926-935, 1972.

[9] B. D. Carlson, "Covariance matrix estimation errors and diagonal loading in adaptive arrays," IEEE Transactions on Aerospace and Electronic Systems, vol. 24, no. 4, pp. 397-401, 1988.

[10] J. Capon, "High resolution frequency-wavenumber spectrum analysis," Proceedings of the IEEE, vol. 57, no. 8, pp. 1408-1418, 1969.

[11] W. A. Gardner, A. Napolitano, and L. Paura, "Cyclostationarity: half a century of research," Signal Processing, vol. 86, no. 4, pp. 639-697, 2006.

[12] Q. Wu and K. M. Wong, "Blind adaptive beamforming for cyclostationary signals," IEEE Transactions on Signal Processing, vol. 44, no. 11, pp. 2757-2767, 1996.

[13] J.-H. Lee and Y.-T. Lee, "Robust adaptive array beamforming for cyclostationary signals under cycle frequency error," IEEE Transactions on Antennas and Propagation, vol. 47, no. 2, pp. 233-241, 1999.

[14] L. C. Godara, "Application of antenna arrays to mobile communications-part II: beam-forming and direction-ofarrival considerations," Proceedings of the IEEE, vol. 85, no. 8, pp. 1195-1245, 1997.

[15] A. Hyvarinen, J. Karhunen, and E. Oja, Independent Component Analysis, John Wiley \& Sons, New York, NY, USA, 2001.

[16] P. Stoica and R. L. Moses, Introduction to Spectral Analysis, Prentice-Hall, Upper Saddle River, NJ, USA, 1997.

[17] P. Stoica, Z. Wang, and J. Li, "Robust Capon beamforming," IEEE Signal Processing Letters, vol. 10, no. 6, pp. 172-175, 2003.

[18] P. Xia and G. B. Giannakis, "Design and analysis of transmitbeamforming based on limited-rate feedback," IEEE Transactions on Signal Processing, vol. 54, no. 5, pp. 1853-1863, 2006.

[19] R. G. Lorenz and S. P. Boyd, "Robust minimum variance beamforming," IEEE Transactions on Signal Processing, vol. 53, no. 5, pp. 1684-1696, 2005.

[20] S. A. Vorobyov, A. B. Gershman, Z.-Q. Luo, and N. Ma, "Adaptive beamforming with joint robustness against mismatched signal steering vector and interference nonstationarity," IEEE Signal Processing Letters, vol. 11, no. 2, part 1, pp. 108-111, 2004. 
[21] T. S. Rappaport, Wireless Communications: Principles and Practice, Prentice-Hall, Upper Saddle River, NJ, USA, 2nd edition, 2002.

[22] A. A. Arowojolu, A. M. D. Turkmani, and J. D. Parsons, "Time dispersion measurements in urban microcellular environments," in Proceedigs of the 44th IEEE Vehicular Technology Conference (VTC'94), vol. 1, pp. 150-154, Stockholm, Sweden, June 1994.

Hui Tong is currently pursuing his Ph.D. degree at Michigan Technological University. His research interests span over the areas of signal processing, information theory, and wireless communications. He has authored more than 15 papers on refereed international journals and conference proceedings. Recently, he focuses on multiantenna channel modeling, channel capacity analysis, and signal processing.

Jafar Pourrostam received the B.S. degree from Amirkabir University of Technology (Tehran Polytechnic), Tehran, Iran, in 2000, and the M.S. degree from University of Tehran, Tehran, Iran, in 2003. He is currently working toward the Ph.D. degree at the Department of Electrical and Computer Engineering, Michigan Technological University, Houghton, MI, USA. His research interests are in digital signal processing,

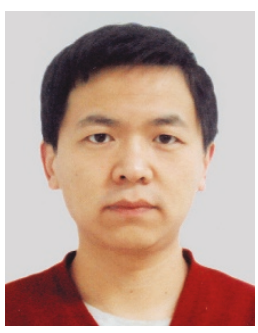
wireless communication systems, and array signal processing.

Seyed A. Zekavat received his Ph.D. from Colorado State University, Fort Collins, Colorado in 2002 in telecommunications. He has published more than 50 journal and conference papers, and has coauthored the book Multi-Carrier Technologies for Wireless Communications, published by Kluwer, an invited chapter in the book Adaptive Antenna Arrays, published by Springer, and an invited paper in Journal of Communications,

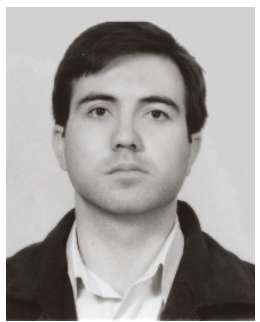
published by Academy Publisher. His research interests are in wireless communications at the physical layer, dynamic spectrum allocation methods, radar theory, blind signal separation and MIMO and beamforming techniques, feature extraction, and neural networking. He is the inventor of wireless local positioning systems (WLPS) with variety of military and civilian applications. He has been awarded by the US NSF Information Technology Research for National Priorities program to study and develop prototypes of WLPS. He is also an active technical program committee member for several IEEE international conferences. At Michigan Tech, he has founded two research laboratories on wireless systems, and is currently principal advisor for several Ph.D. students. 